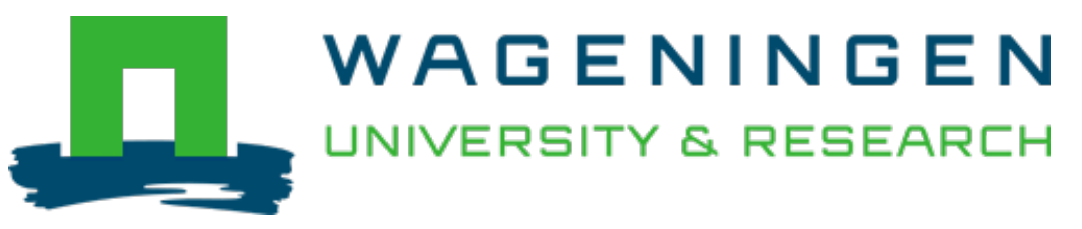

\title{
Characterization of Fen-Daqu Through Multivariate Statistical Analysis of H-1 NMR Spectroscopic Data
}

\author{
Journal of the Institute of Brewing \\ Van-Diep, L.; Zheng, X.; Ma, K.; Chen, J.Y.; Han, B.Z. et al \\ https://doi.org/10.1002/j.2050-0416.2011.tb00499.x
}

This publication is made publicly available in the institutional repository of Wageningen University and Research, under the terms of article $25 \mathrm{fa}$ of the Dutch Copyright Act, also known as the Amendment Taverne. This has been done with explicit consent by the author.

Article 25 fa states that the author of a short scientific work funded either wholly or partially by Dutch public funds is entitled to make that work publicly available for no consideration following a reasonable period of time after the work was first published, provided that clear reference is made to the source of the first publication of the work.

This publication is distributed under The Association of Universities in the Netherlands (VSNU) 'Article $25 \mathrm{fa}$ implementation' project. In this project research outputs of researchers employed by Dutch Universities that comply with the legal requirements of Article $25 \mathrm{fa}$ of the Dutch Copyright Act are distributed online and free of cost or other barriers in institutional repositories. Research outputs are distributed six months after their first online publication in the original published version and with proper attribution to the source of the original publication.

You are permitted to download and use the publication for personal purposes. All rights remain with the author(s) and / or copyright owner(s) of this work. Any use of the publication or parts of it other than authorised under article $25 \mathrm{fa}$ of the Dutch Copyright act is prohibited. Wageningen University \& Research and the author(s) of this publication shall not be held responsible or liable for any damages resulting from your (re)use of this publication.

For questions regarding the public availability of this publication please contact openscience.library@wur.nl 


\title{
Characterization of Fen-Daqu Through Multivariate Statistical Analysis of ${ }^{1} \mathrm{H}$ NMR Spectroscopic Data
}

\author{
L. Van-Diep ${ }^{1}$, X-.W. Zheng ${ }^{1,3}$, K. Ma ${ }^{1}$, J-.Y. Chen ${ }^{1}$, B-.Z. Han ${ }^{1, *}$ and M. J. R. Nout ${ }^{3}$
}

\begin{abstract}
J. Inst. Brew. 117(4), 516-522, 2011

Fen liquor is typical of Chinese light-flavour liquor (alcoholic spirit), which is fermented from sorghum with Fen-Daqu powder. Fen-Daqu is a saccharifying agent and fermentation starter in this fermentation process and in Fen traditional vinegar. To investigate the changes of biochemical components in Fen-Daqu during the incubation, samples at seven incubation stages were analyzed by ${ }^{1} \mathrm{H}$ nuclear magnetic resonance (NMR) spectrometry and principal component analysis (PCA). This revealed clear separation of the samples obtained from different incubation stages in the principal component plots by combining PC1 and PC2, which cumulatively accounted for $93.27 \%$ of the variance. The major compounds that contributed to discrimination were acetate/alanine, arginine, ascorbate, betaine, choline, ethanol, fructose, galactose, glucose, glucitol, glycerate, lactate, maltose, mannitol, phenylalanine, proline, propylene glycol, threonine and tryptophan. These compounds were regarded as the representative metabolites or biomarkers characteristic for each incubation stage and were related with microbiological changes of importance for quality control in Fen-Daqu production.
\end{abstract}

Key words: Fen-Daqu, fermentation starter, ${ }^{1} \mathrm{H}$ nuclear magnetic resonance, principal component analysis.

\section{INTRODUCTION}

Fen liquor is light-flavoured, and is one of the famous Chinese liquors (alcoholic spirit) with a history of over 1,500 years. Fen liquor is fermented and distilled from sorghum with Fen-Daqu powder made from barley and pea. Daqu is a saccharifying agent and fermentation starter for the production of Chinese liquor, and contains a range of microorganisms, various enzymes, metabolites and degradation products, and important flavour compounds that will contribute to the aroma of the final distillate $e^{3,8,14}$. According to the approximate temperature and level of cooling during the incubation step, Fen-Daqu can

\footnotetext{
${ }^{1}$ College of Food Science and Nutritional Engineering, China Agricultural University, Beijing 100083, China.

${ }^{2}$ Faculty of Agriculture Forestry Fishery, Vinh University, Vinh City, Nghe An Province 42000, Vietnam.

${ }^{3}$ Laboratory of Food Microbiology, Wageningen University, P.O. Box 8129, 6700 EV Wageningen, The Netherlands.

*Corresponding author. E-mail: hbz@ cau.edu.cn
}

Publication no. G-2011-1128-1147

(c) 2011 The Institute of Brewing \& Distilling be classified into three types as follows: (i) Fen-Houhuo, high-temperature and medium cooling (ii) Fen-Hongxin, long high-temperature and low cooling; (iii) Fen-Qingcha, medium temperature and high cooling, and their proportion in Fen liquor production is 40\%:30\%:30\%. Fen-Daqu is prepared from barley and peas by five steps: (1) ingredient formulation; (2) grinding and mixing; (3) shaping; (4) incubation (about 1 month) and (5) maturation (about 6 months). The incubation step is divided into seven stages: Woqu, Shangmei, Liangmei, Chaohuo, Dahuo, Houhuo and Yangqu. Woqu is the first day of incubation and the Daqu is still quite soft and thus may only be stacked to a maximum of three layers, to avoid deformation and allow good ventilation. Shangmei means "growth of filamentous fungal mycelium". Fungi occur as a natural inoculum on raw materials, water, rush mats, bran coats and in the environment. The temperature increases gradually, attaining $30-40^{\circ} \mathrm{C}$ in $2-3$ days. Liangmei means a cooling down to prevent damage from overheating and a hardening of the Daqu, the temperature is around 25$35^{\circ} \mathrm{C}$ for $2-3$ days. The key incubation period for microbial succession is "Chaohuo", the temperature increases to about $43-47^{\circ} \mathrm{C}$ and this stage takes about 5-6 days. The Dahuo stage takes about 4-6 days and the temperature reaches a maximum of around $52^{\circ} \mathrm{C}$. The Houhuo stage takes about 5-6 days and the temperature decreases gradually to approximately $30-35^{\circ} \mathrm{C}$. Yangqu (pre-maturation) takes about 5-7 days and the aim of this stage is to allow the equilibration of moisture, acidity and enzyme activity $^{18}$.

There have been a few studies on the end product of sauce-flavour, light-flavour and strong-flavour Daqu. The results showed that many biochemical compounds in Daqu served as flavour compounds or flavour precursors in the liquor obtained by this type of $D a q u^{3,14,17}$. We hypothesize that the profile of the biochemical characteristics in Daqu during the incubation period can reflect the specific fermentation events and biochemical reactions associated with the microbial succession and metabolism taking place during its stages. However, up to now, no biochemical characteristics of Fen-Daqu during the various stages of incubation have been reported.

Nuclear magnetic resonance (NMR) is one of the important non-targeted techniques. It can produce rapid, nondestructive, stable and highly reproducible results ${ }^{5}$. It has the advantage that all kinds of small metabolite molecules can be measured simultaneously ${ }^{1,9}$. Principal component analysis (PCA) is a multivariate statistical analysis 
technique that is used for data reduction. Metabolomic techniques combining NMR and PCA have been applied to the metabolic profiling of various kinds of Daqu products, fermented soybean, wine, beer and cheese $e^{2,4,6,11,13,14,16}$.

In this study, we used ${ }^{1} \mathrm{H}$ NMR spectroscopy followed by PCA for the analysis of the biochemical compounds from extracts of Fen-Daqu samples obtained during the successive stages of the incubation steps. The objective of the study was to reveal the major components of Fen$D a q u$, and also to assess whether these components could be used to discriminate Fen-Daqu intermediate products obtained during the successive stages of incubation.

\section{MATERIALS AND METHODS}

\section{Sampling}

Fen-Daqu samples were obtained from the Xinghuacun Fenjiu Group, Shanxi province, China. Daqu was fermented and matured in stacked layers. Samples were collected at the end of Woqu, Shangmei, Liangmei, Chaohuo, Dahuo, Houhuo and Yangqu stages of Fen-Houhuo, FenHongxin and Fen-Qingcha. Each sample was obtained by randomly selecting from each upper, middle and lower stacked layer and mixed together as an experimental sample. Samples were stored at $-80^{\circ} \mathrm{C}$ until used.

\section{Extraction of polar compounds from Fen-Daqu}

One hundred milligrams of ground Daqu sample was transferred into a centrifuge tube and then $1.5 \mathrm{~mL}$ of cold Milli-Q water $\left(\sim 0^{\circ} \mathrm{C}\right.$, ice bath) was added into the tube and vortexed at 2,500 oscillations/min for $60 \mathrm{sec}$ using a Biospec Beadbeater (Mini-Beadbeater-8, Biospec, Bartlesville, USA) without added beads. The tube was then kept on ice for $10 \mathrm{~min}$ and then centrifuged for $10 \mathrm{~min}$ at $16,060 \times g$ at $4^{\circ} \mathrm{C}$. Finally, one millilitre of supernatant was transferred to a new tube and stored at $-80^{\circ} \mathrm{C}$ until analysis, as described previously ${ }^{14}$. Each experiment was performed in triplicate.

\section{NMR measurements}

For the NMR measurements, each extract was mixed with an equal volume of cold loading buffer $(0.1 \mathrm{M}$ of sodium phosphate, $\mathrm{pH} 7.0$, containing $10 \% \mathrm{D}_{2} \mathrm{O}(\mathrm{v} / \mathrm{v}), 1$ $\mathrm{mM}$ TSP (3-trimethylsilylpropionate) as an internal standard, $100 \mathrm{mM}$ imidazole and $0.2 \% \mathrm{w} / \mathrm{v}$ sodium azide) and centrifuged at $16,060 \times g$ at $4^{\circ} \mathrm{C}$. A $500 \mu \mathrm{L}$ aliquot of the mixture was transferred into a $5 \mathrm{~mm}$ NMR tube for determination. All ${ }^{1} \mathrm{H}$ NMR spectra were measured at $300 \mathrm{~K}$ using an Avance III NMR spectrometer (Bruker, Germany, proton frequency $600.13 \mathrm{MHz}, 14.1 \mathrm{~T}$ ). For each sample, 128 scans were recorded with the following parameters: pulse sequence, noesygppr1d; relaxation delay, $4 \mathrm{sec}$; mixing time (for noesy), 1s; acquisition time, 2.28 $\mathrm{s}$; number of steady states transients (dummy scans), 4; gradient pulse times, $1 \mathrm{~ms}$; solvent suppression, presaturation with spoil gradient; spectral width, 7,184 Hz; time domain size, $32 \mathrm{k}$.

\section{Data analysis}

The spectral region from $0.60 \mathrm{ppm}$ to $9.00 \mathrm{ppm}$ was segmented into regions of $0.04 \mathrm{ppm}$ width giving a total of 210 integrated regions per NMR spectrum. The region from 4.40 to 5.30 was excluded from the analysis because of the residual signal of water in the aqueous extracts, whereas those from 7.35 to 7.50 and 8.40 to 8.60 were excluded from the analysis because the residual signal of imidazole. All spectral data were scaled to total intensity

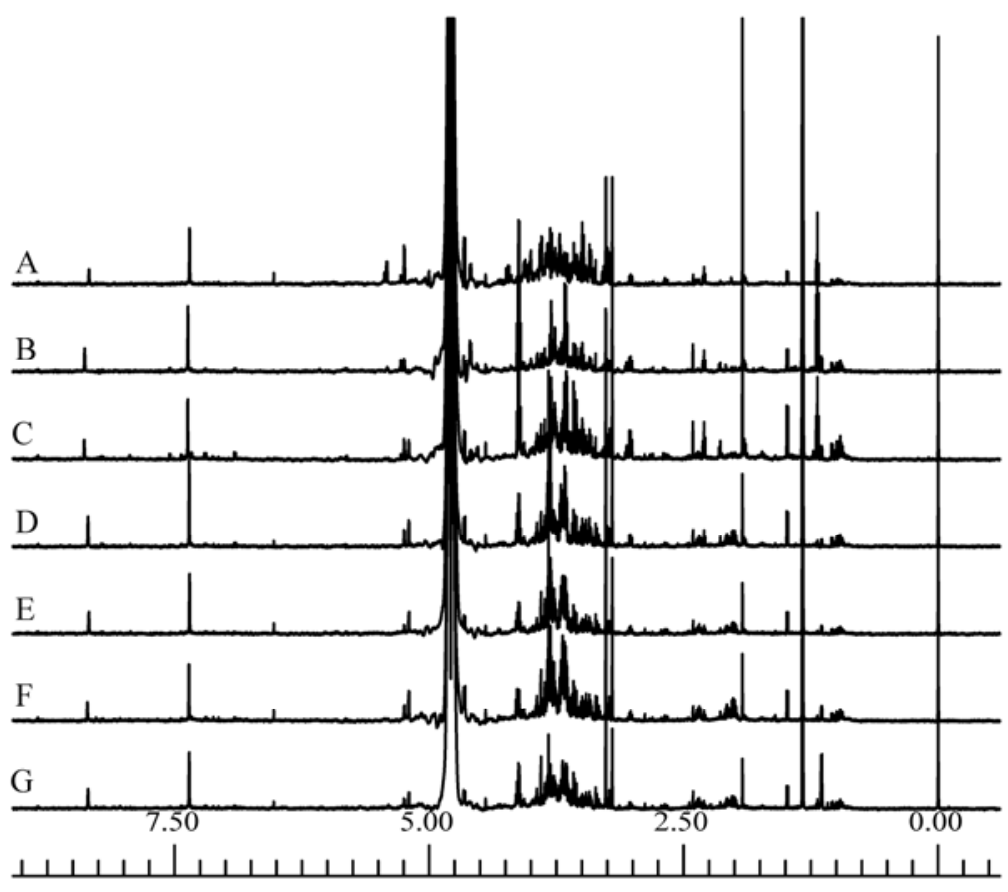

Fig. 1. Representative ${ }^{1} \mathrm{H}$ NMR spectra of aqueous extracts of Fen-Daqu, obtained from (A) Woqu, (B) Shangmei, (C) Liangmei, (D) Chaohuo, (E) Dahuo, (F) Houhuo, and (G) Yangqu. 
Table I. Presumptive biochemical compounds and their concentrations in Fen-Daqu. Values are in micromoles per gram of Daqu dry matter by means of Chenomx.

\begin{tabular}{|c|c|c|c|c|c|c|c|}
\hline Metabolite & Woqu & Shangmei & Liangmei & Chaohuo & Dahuo & Houhuo & Yangqu \\
\hline 1,6-Anhydro- $\beta$-D-glucose & 2.20 & 1.28 & 1.92 & 1.21 & 1.50 & 1.60 & 0.86 \\
\hline 2-Aminobutyrate & 0.87 & 0.27 & 0.63 & 1.49 & 0.61 & 1.43 & 0.54 \\
\hline 2-Hydroxyglutarate & 0.98 & $\mathrm{nd}^{\mathrm{a}}$ & nd & 1.01 & 2.04 & 0.66 & 1.06 \\
\hline 4-Aminobutyrate & 4.59 & 3.69 & 6.60 & 4.85 & 2.85 & 3.58 & 0.55 \\
\hline 4-Carboxyglutamate & nd & 0.85 & 1.37 & 1.18 & 2.40 & 1.85 & 1.71 \\
\hline Acetate & 7.02 & 51.10 & 32.85 & 9.04 & 13.27 & 6.47 & 5.78 \\
\hline Acetoacetate & 1.31 & 0.69 & 0.85 & 0.73 & 0.68 & 1.42 & 0.58 \\
\hline Adenosine & 0.71 & nd & 0.38 & 0.46 & 0.26 & 0.47 & 0.71 \\
\hline Alanine & 4.69 & 5.28 & 12.15 & 9.74 & 8.55 & 7.57 & 5.12 \\
\hline Alloisoleucine & 0.38 & 0.35 & 0.73 & 0.58 & 0.42 & 0.80 & 0.25 \\
\hline Anserine & 0.83 & 0.82 & 0.70 & 1.08 & 1.17 & 1.20 & 0.43 \\
\hline Arginine & 3.01 & 1.74 & 2.79 & 3.01 & 0.75 & 3.23 & 1.51 \\
\hline Ascorbate & 3.36 & 1.66 & 3.54 & 1.85 & 2.75 & 2.15 & 2.22 \\
\hline Asparagine & 1.64 & 1.08 & 0.79 & 1.29 & 0.81 & 1.86 & 0.38 \\
\hline Aspartate & 2.74 & 1.06 & 3.15 & 2.72 & 1.46 & 1.35 & 0.50 \\
\hline Betaine & 4.33 & 2.57 & 5.00 & 5.97 & 6.09 & 5.42 & 8.39 \\
\hline Caffeine & 0.20 & 0.09 & 0.43 & 0.26 & 0.27 & 0.19 & 0.18 \\
\hline Carnitine & 0.28 & 0.14 & 0.69 & 0.56 & 0.63 & 0.49 & 0.31 \\
\hline Choline & 6.32 & 3.67 & 4.78 & 4.79 & 4.68 & 4.16 & 3.55 \\
\hline Creatinine & 0.64 & 0.17 & 0.24 & 0.27 & 0.33 & 0.14 & 0.15 \\
\hline Cystine & 1.48 & nd & nd & 1.44 & 1.05 & 1.28 & 0.95 \\
\hline Dimethylamine & nd & nd & 0.07 & 0.15 & 0.21 & 0.22 & 0.16 \\
\hline Ethanol & 11.31 & 43.89 & 22.97 & 6.20 & 3.41 & 1.78 & 4.46 \\
\hline Fructose & 27.44 & 5.74 & 3.72 & 4.30 & 3.25 & 3.35 & 2.96 \\
\hline Fucose & 0.48 & 1.00 & 0.78 & 0.49 & 0.29 & 0.41 & 0.50 \\
\hline Fumarate & 2.82 & 0.08 & 0.26 & 0.97 & 2.35 & 1.25 & 1.16 \\
\hline Galactitol & 2.26 & 0.74 & 2.04 & 1.82 & 1.99 & 2.33 & 1.83 \\
\hline Galactonate & 2.75 & 0.75 & 1.36 & 1.68 & 1.97 & 1.72 & 2.40 \\
\hline Galactose & 10.50 & 12.24 & 11.44 & 10.22 & 4.42 & 6.71 & 5.26 \\
\hline Glucitol & 4.75 & 2.46 & 3.35 & 5.11 & 3.83 & 4.54 & 3.84 \\
\hline Gluconate & 5.28 & 2.19 & 4.09 & 3.20 & 2.32 & 2.84 & 2.18 \\
\hline Glucose & 60.72 & 14.26 & 23.04 & 19.91 & 19.61 & 19.96 & 8.63 \\
\hline Glutamate & 2.14 & 2.51 & 5.82 & 5.33 & 4.74 & 5.26 & 4.00 \\
\hline Glutamine & 1.31 & 1.16 & 1.82 & 1.67 & 1.38 & 1.52 & 0.84 \\
\hline Glutaric acid monomethyl ester & 0.23 & 0.39 & 0.28 & 0.56 & 0.54 & 0.36 & 0.35 \\
\hline Glycerate & 4.67 & 2.46 & 5.05 & 3.53 & 3.90 & 4.14 & 2.73 \\
\hline Glycerol & 5.91 & 9.88 & 18.90 & 11.81 & 11.34 & 9.12 & 10.08 \\
\hline Glycine & 3.21 & 3.30 & 5.10 & 4.36 & 3.77 & 3.35 & 2.19 \\
\hline \multicolumn{8}{|c|}{ (continued on next page) } \\
\hline
\end{tabular}

a Not detected.

of the corresponding spectrum, and then analyzed by principal component analysis (PCA) with Pareto scaling. PCA was performed with AMIX software (version 3.7.10; Bruker BioSpin, Rheinstetten, Germany). The output from the PCA analysis consisted of score plots giving an indication of the differentiation of the classes in terms of metabolome similarity and loading plots giving an indication as to which NMR spectral regions were important with respect to the classification obtained in the score plots. Compounds were identified and quantified with Chenomx software (Version 6.0; Chenomx, Edmonton, Canada).

\section{RESULTS AND DISCUSSION}

\section{Visual inspection of ${ }^{1} \mathrm{H}$ NMR spectra and assignment of compounds}

Representative ${ }^{1} \mathrm{H}$ NMR spectra of aqueous extracts of Fen-Daqu during its production are presented in Fig. 1. A wide range of biochemical compounds could be assigned in each spectral matrix that provided complementary information on overall changes of the biochemical compounds during the production of Fen-Daqu. About 80 biochemical compounds were presumptively identified by Chenomx software 6.0 as shown in Table I.
In sample preparation, the extracts of Fen-Daqu contained added imidazole and sodium azide, these compounds were not added in the previous study ${ }^{14}$. Imidazole was used as $\mathrm{pH}$ indicator and the $\mathrm{pH}$ values of samples were in the range 6.68-7.05. Sodium azide was used to inhibit the growth of bacteria.

Table I showed that the amounts of sugars such as glucose, fructose, maltose, sucrose, ribose and lactose gradually decreased during incubation. Obviously these decreases reflect the assimilation of carbon as a source of energy by the evolving microbiota. Such microbiological developments in Fen-Daqu were reported previously ${ }^{7}$. In addition, it was reported that fungi had a unique distribution in the starter of Fen liquor. They possessed high $\alpha-$ amylase and glucoamylase activities, which enabled the utilization of raw starch as a carbon source ${ }^{12}$.

Table I also shows that the concentration of proline, homoserine, tyrosine and threonine were lower during the early stages than during the later stage of production. During the middle stage, especially the Dahuo stage, the temperature in the Daqu blocks was increased to $52^{\circ} \mathrm{C}$. It had been stated that the goal of the Dahuo stage is to enhanced proteolysis and the accumulation of amino acids ${ }^{18}$. During the middle stage, the concentrations of acetate, 
Table I. (continued from previous page).

\begin{tabular}{|c|c|c|c|c|c|c|c|}
\hline Metabolite & Woqu & Shangmei & Liangmei & Chaohuo & Dahuo & Houhuo & Yangqu \\
\hline Glycylproline & 2.93 & 2.12 & 2.47 & 3.12 & 1.94 & 3.71 & 2.59 \\
\hline Guanidoacetate & 5.51 & 4.15 & 3.62 & 7.25 & 6.92 & 5.52 & 2.02 \\
\hline Histamine & 1.29 & 0.69 & 1.30 & 0.53 & 0.87 & 0.79 & 0.67 \\
\hline Histidine & 0.95 & 0.63 & 1.01 & 0.69 & 1.90 & 0.63 & 1.08 \\
\hline Homocysteine & 2.31 & nd & 0.85 & 0.41 & 0.53 & 1.10 & nd \\
\hline Homoserine & 1.50 & 2.21 & 2.82 & 2.32 & 3.54 & 3.49 & 2.62 \\
\hline Isoleucine & 0.55 & 0.62 & 1.86 & 1.46 & 1.21 & 1.69 & 0.72 \\
\hline Isopropanol & 1.31 & 0.99 & 1.01 & nd & 0.47 & 1.22 & 1.55 \\
\hline Lactate & 1.41 & 115.83 & 139.58 & 103.50 & 66.39 & 55.45 & 28.76 \\
\hline Lactose & 4.22 & 2.39 & 3.81 & 2.98 & 2.38 & 1.73 & 1.28 \\
\hline Leucine & 0.93 & 1.58 & 2.97 & 2.73 & 2.56 & 2.70 & 0.93 \\
\hline Lysine & 0.82 & 2.28 & 3.28 & 2.94 & 2.86 & 1.77 & 0.42 \\
\hline Malate & 5.14 & 0.80 & 0.73 & 3.04 & 2.08 & 3.75 & 2.72 \\
\hline Malonate & 0.56 & nd & nd & 0.43 & 0.47 & 0.34 & 0.40 \\
\hline Maltose & 9.48 & 2.79 & 1.74 & 2.03 & 2.08 & 2.49 & 1.39 \\
\hline Mannitol & 3.57 & 7.79 & 10.54 & 6.27 & 10.07 & 6.38 & 2.78 \\
\hline Mannose & 3.09 & 2.06 & 5.70 & 4.82 & 5.46 & 4.00 & 4.21 \\
\hline Methanol & 3.50 & 3.77 & 3.82 & 2.69 & 3.64 & 5.27 & 2.42 \\
\hline Methionine & 0.28 & 0.51 & 1.27 & 0.81 & 0.60 & 0.66 & 0.38 \\
\hline myo-Inositol & 6.72 & 2.46 & 3.35 & 2.36 & 1.72 & 2.35 & 1.29 \\
\hline O-Phosphocholine & 1.10 & 0.39 & 1.33 & 1.08 & 0.63 & 0.21 & 0.24 \\
\hline Ornithine & nd & 1.71 & 1.72 & 0.93 & 0.45 & nd & 0.53 \\
\hline Phenylalanine & 0.30 & 0.41 & 2.35 & 0.18 & 2.49 & 1.21 & 0.40 \\
\hline Proline & 1.70 & 2.32 & 6.11 & 17.78 & 16.20 & 16.53 & 13.16 \\
\hline Propylene glycol & 0.25 & 1.35 & 2.50 & 0.94 & 0.78 & 1.47 & 2.53 \\
\hline Pyruvate & 0.67 & nd & 0.76 & 0.34 & 0.60 & 1.18 & 0.55 \\
\hline Ribose & 15.19 & 4.67 & 6.80 & 7.00 & 7.64 & 3.99 & 3.13 \\
\hline Saccharopine & nd & 0.31 & 1.16 & 1.58 & nd & 0.86 & nd \\
\hline Succinate & 0.60 & 2.41 & 2.80 & 1.69 & 1.59 & 1.38 & 0.97 \\
\hline Sucrose & 3.19 & 1.16 & 1.25 & 0.82 & 0.28 & 1.10 & 1.42 \\
\hline Taurine & 2.46 & 1.20 & 2.06 & 0.94 & 3.11 & 3.97 & 1.98 \\
\hline Threonine & 0.99 & 2.81 & 3.09 & 3.98 & 2.11 & 2.26 & 3.69 \\
\hline Trigonelline & 2.15 & nd & 2.39 & nd & 1.31 & 2.21 & 0.86 \\
\hline Trimethylamine $\mathrm{N}$-oxide & 0.91 & 0.20 & 0.60 & 0.65 & 0.62 & 1.63 & 0.34 \\
\hline Tryptophan & 0.65 & 1.52 & 1.96 & 2.04 & 0.80 & 1.06 & 0.63 \\
\hline Tyramine & nd & 0.17 & 0.96 & 0.51 & 0.08 & 0.27 & 0.28 \\
\hline Tyrosine & 0.23 & 1.09 & 1.34 & 0.78 & 1.65 & 1.51 & 0.33 \\
\hline Uracil & 0.74 & 1.64 & 2.01 & 1.45 & 0.71 & 1.05 & 0.64 \\
\hline Valine & 1.48 & 1.77 & 3.56 & 2.58 & 2.36 & 1.70 & 0.95 \\
\hline Xylose & 1.96 & nd & 5.67 & 3.64 & 1.51 & nd & 2.50 \\
\hline$\pi$-Methylhistidine & 1.29 & nd & 0.55 & 0.97 & 1.68 & 0.84 & 1.30 \\
\hline
\end{tabular}

alanine, lactate, leucine, glycine, glycerol, mannitol and valine were relatively higher than during the early and later stages, as shown in Table I. Lactate is produced by lactic acid bacteria ${ }^{10}$. The changes in lactate concentration showed a positive correlation with the changes in the lactic acid bacteria concentration during these stages. The experimental results showed that the lactic acid bacteria counts were highest at during the Shangmei, Liangmei and Chaohuo stages, and were in the range of 5.7-6.4 Log $\mathrm{CFU} \mathrm{g}{ }^{-1}$. However, lactate was not detected in the end product of light-flavour $D a q u^{14}$, so it could be that the lactate was reduced during the maturation step.

According to the flavour characteristics of the liquor obtained, several types of Daqu can be distinguished, such as light-flavoured, strong-flavoured, and sauce-flavoured Daqu. It has been reported that glycerol, acetate, carnitine and malate were identified as biomarkers of light-flavoured $D a q u^{14}$. Table I shows that the concentration of glycerol, acetate and malate were relatively higher during the incubation step. Acetate was also reported as the representative aroma compound in light-flavoured liquor, and mainly as ethyl acetate ${ }^{15}$. Ethyl acetate and 3methyl-1-butanol acetate were found at relatively high concentrations in the Shangmei and Liangmei stages (data not shown).

\section{Principal component analysis}

PCA was performed encompassing all of the samples and enabled a discrimination among samples. The PCA scores plot (Fig. 2) showed that, samples collected at each stage of the production of Fen-Daqu could be discriminated clearly. The first two principal components (PC1 and PC2) cumulatively accounted for $93.27 \%$ of the total variation. Separation of the different samples of Fen-Daqu in the score plot was achieved by combining PC1 and PC2. In the score plot, samples obtained from the Woqu, Shangmei and Liangmei stages were separated clearly from each other. The score plots for the samples obtained from Liangmei, Chaohuo and Dahuo stages were relatively close to each other, but were nevertheless separated (Fig. 4c and 4d). Similarly, score plots for the samples obtained from the Dahuo, Ноиhиo and Yangqu stages were separated clearly from each other. The distances between the score plots of the incubation stages indicate that the metabolite profiles undergo the biggest changes 
from A (Woqu) to B (Shangmei) stage and from the $\mathrm{E}$ (Dahuo) to F (Houhuo) stage.

The biochemical compounds in the aqueous extracts of Fen-Daqu could be distinguished using PCA, and is

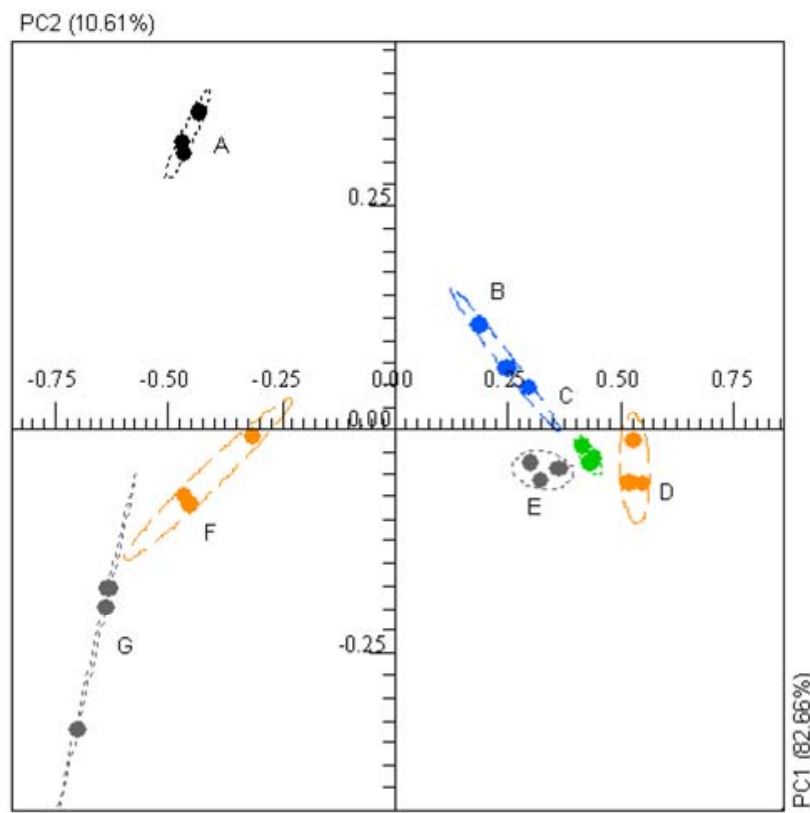

Fig. 2. PCA score plot of Fen-Daqu during the incubation (A) Woqu, (B) Shangmei, (C) Liangmei, (D) Chaohuo, (E) Dahuo, (F) Houhuo, and (G) Yangqu. shown in the loading plot for PC1 and PC2 (Fig. 3). The major compounds that contributed to discrimination were acetate/alanine, arginine, ascorbate, betaine, choline, ethanol, fructose, galactose, glucose, glucitol, glycerate, homoserine, lactate, maltose, mannitol, phenylalanine, propylene glycol, threonine and tryptophan. Of these compounds, acetate/arginine, fructose, galactose, glucose, glucitol, lactate, maltose, mannitol, phenylalanine, threonine and tryptophan contributed mainly to discrimination by $\mathrm{PC} 1$, while alanine/ascorbate, betaine, choline, ethanol, glucose, glycerate, homoserine, lactate, maltose, propylene glycol, threonine and tryptophan contributed mainly to discrimination by PC2.

The biomarkers of each incubation stage were identified by pairwise comparison of peak intensities among the seven stages. Figure 4 shows the pairwise PCA comparisons of each incubation stage of Fen-Daqu. All score plots displayed a significant separation between the respective stages of the production process. The loading plots indicate the biochemical compounds that were responsible for the separation of clusters.

As shown in Fig. 4a, the score plot shows a separation between the Woqu and Shangmei stages. Loadings plot shows that the biochemical compounds responsible for the separation were increased in the concentrations of acetate, lactate, glycerol, ethanol, mannitol, homoserine, phenylalanine, propylene glycol and decreased in the concentrations of choline, betaine, glucose, fructose, aspartate, maltose, sucrose and taurine. Figure $4 \mathrm{~b}$ shows the separations of the samples obtained from the Shangmei and Liangmei

(a)

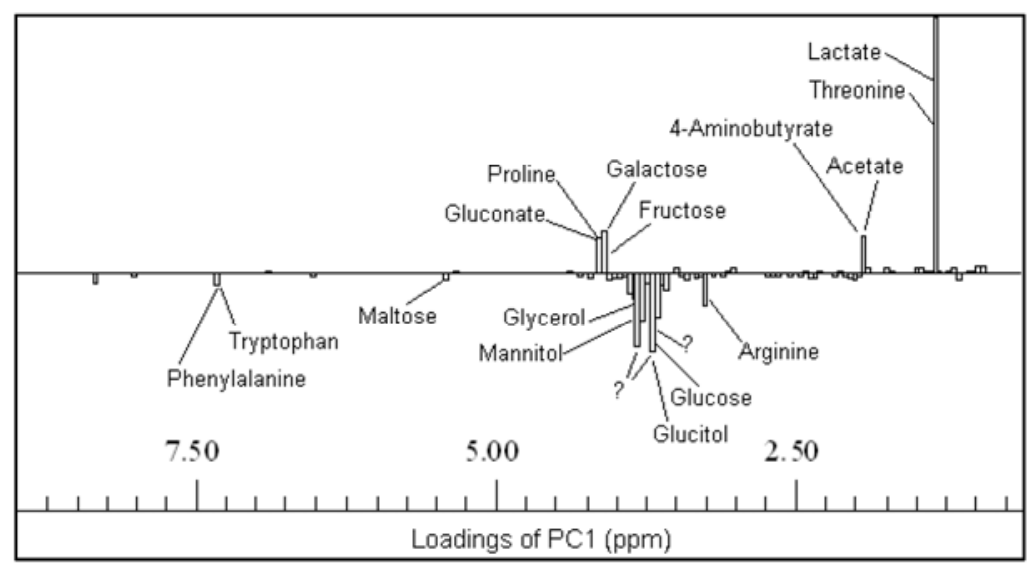

(b)

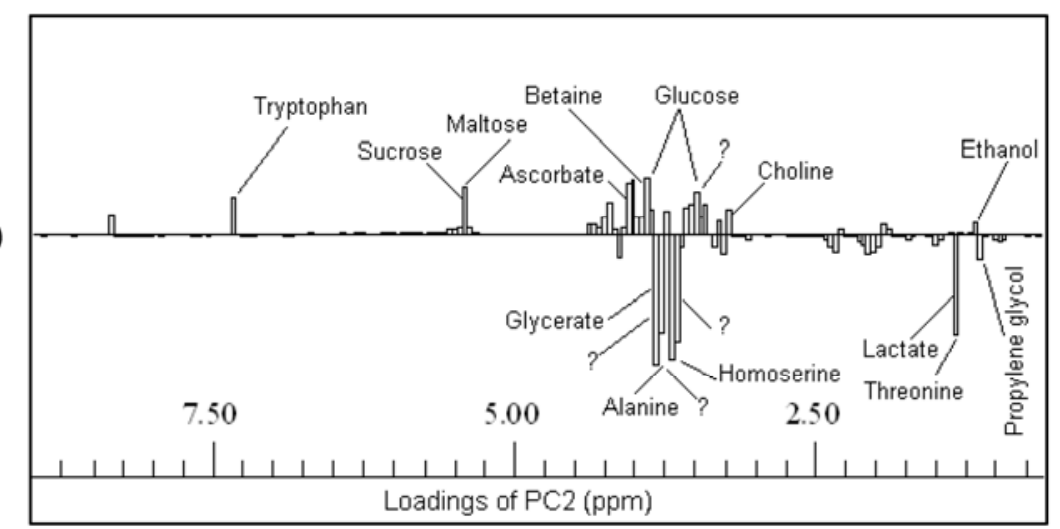

Fig. 3. PCA loading plot for PC1 (a) and PC2 (b) of Fen-Daqu during the incubation. 

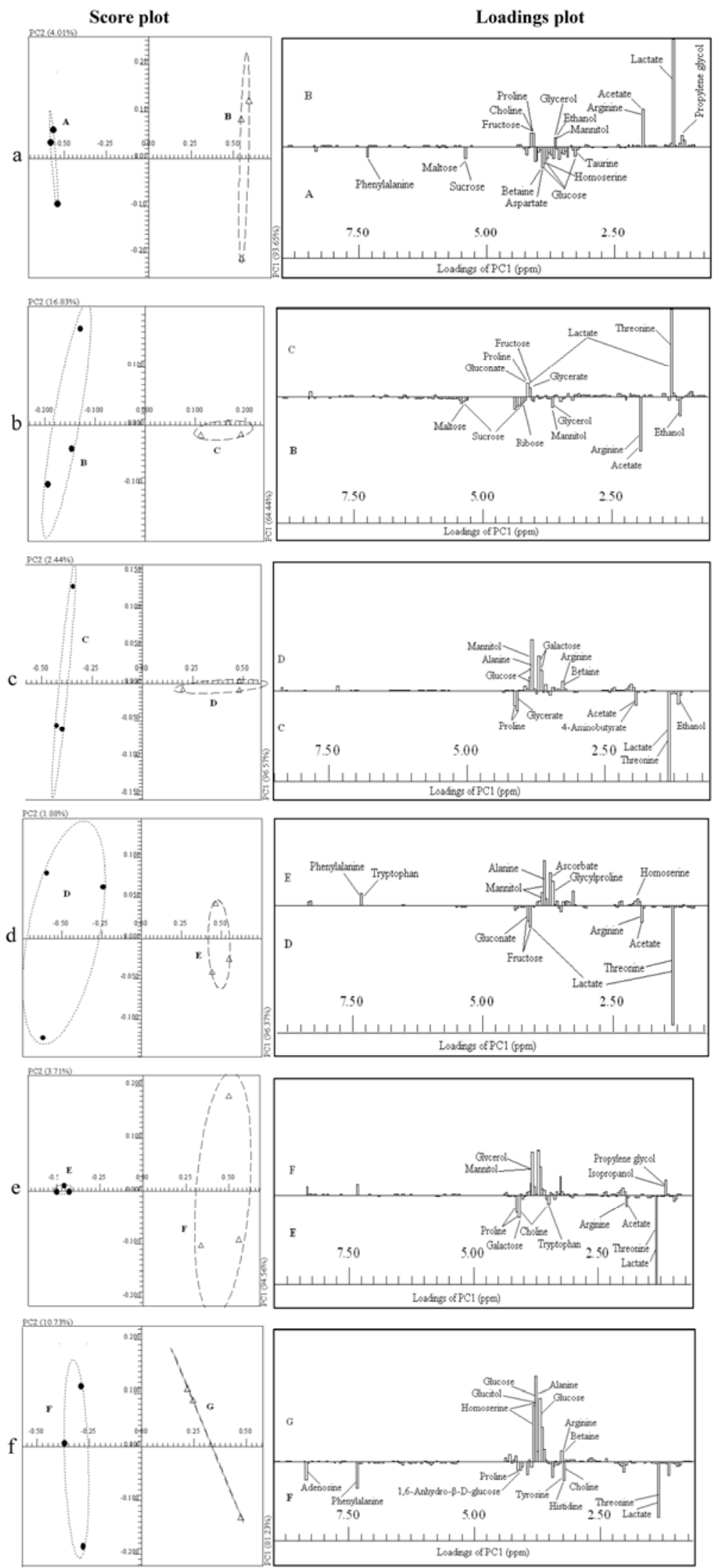

Fig. 4. PCA of Fen-Daqu extracts of incubation stages: (a) A: Woqu versus B: Shangmei, (b) B: Shangmei versus C: Liangmei, (c) C: Liangmei versus D: Chaohuo, (d) D: Chaohuo versus E: Dahuo, (e) E: Dahuo versus F: Houhuo, and (f) F: Houhuo versus G: Yangqu. 
stages; these were caused by increases in the concentration of lactate, arginine, gluconate, glycerate, glycerol, proline, mannitol, ribose, sucrose, threonine and decreases in the concentration of acetate, ethanol, maltose, and fructose. Figure $4 \mathrm{c}$ shows the separations of the samples obtained from the Liangmei and Chaohuo stages that were caused by increases in the concentrations of lactate, arginine, proline, threonine, and decreases in the concentrations of 4-aminobutyrate, galactose, glycerate, glucose, acetate, betaine, alanine, ethanol and mannitol. Figure $4 d$ shows the separations of the samples obtained from the Chaohuo and Dahuo stages that were caused by increases in the concentrations of acetate, ascorbate, homoserine, phenylalanine, and mannitol and decreases in the concentrations of alanine, arginine, lactate, gluconate, glycylproline, threonine, and tryptophan. Figure $4 \mathrm{e}$ shows the separations of the samples obtained from the Dahuo and Houhuo stages; these were caused by increases in the concentrations of arginine, galactose, isopropanol, proline, propylene glycol, threonine, tryptophan and decreases in the concentrations of acetate, choline, glycerol, lactate, and mannitol. Similarly, Fig. 4f shows a clear separation between the Houhuo and Yangqu stage. Loadings plots show that the biochemical compounds responsible for the separation were increased concentrations of betaine, histidine, adenosine, threonine, and decreased concentrations of alanine, arginine, choline, homoserine, glucitol, lactate, phenylalanine, proline, tyrosine, and 1,6-anhydro- $\beta$-Dglucose. The absolute concentrations of all biochemical compounds are presented in Table I.

To this day, the manufacturing process of Fen liquor still relies on workers' experience. The production of FenDaqu is still the constitution of the traditional fermentation technology without added microorganisms. The quality of Daqu cannot be kept stable, even for the same batch of products. This study is the first joint application of ${ }^{1} \mathrm{H}$ NMR to investigate the changes in the biochemical components in Fen-Daqu during the incubation step. Having gained this important insight into the changes of biochemical components of Fen-Daqu, we potentially have the knowledge to make substantial improvements to the liquor fermentation process, such as improving the beneficial strains, and so further study of microbial ecology during the incubation step of Fen-Daqu will be necessary.

In conclusion, the changes in the biochemical compounds in Fen-Daqu during the incubation step were revealed in this study. This could help Daqu producers monitor the progress of the Daqu manufacturing process, by measuring specific biomarkers for each stage, and to verify the authenticity of commercially produced Fen-Daqu.

\section{ACKNOWLEDGEMENTS}

We thank Mr. Hu Gao-Fei for NMR support and the Shanxi Xinghuacun Fenjiu Group Company for Daqu sampling assistance and advice. This study was funded by the National Natural Science Foundation of China (No. 31071592) and the KNAWChina Joint Research Project (No. 07CDP015) from the Royal Netherlands Academy of Arts and Sciences.

\section{REFERENCES}

1. Choi, H. K., Yoon, J. H., Kim, Y. S. and Kwon, D. Y., Metabolomic profiling of Cheonggukjang during fermentation by
${ }^{1} \mathrm{H}$ NMR spectrometry and principal components analysis. Process Biochem., 2007, 42, 263-266.

2. Duarte, I., Barros, A., Belton, P. S., Righelato, R., Spraul, M., Humpfer, E. and Gil, A. M., High-resolution nuclear magnetic resonance spectroscopy and multivariate analysis for the characterization of beer. J. Agric. Food Chem., 2002, 50, 2475-2481.

3. Fan, W. L., Zhang, Y. H. and Xu, Y., Analysis of volatile microconstituents in Daqu by HS-SPME and GC-MS. Liquor-making Sci. Technol., 2007, 12(162), 74-78 (in Chinese).

4. Lachenmeier, D. W., Frank, W., Humpfer, E., Schäfer, H., Keller, S., Mörtter, M. and Spraul, M., Quality control of beer using highresolution nuclear magnetic resonance spectroscopy and multivariate analysis. Eur. Food Res. Technol., 2005, 220, 215-221.

5. Lachenmeier, D. W., Humpfer, E., Fang, F., Schütz, B., Dvortsak, P., Sproll, C. and Spraul, M., NMR-spectroscopy for nontargeted screening and simultaneous quantification of health-relevant compounds in foods: the example of melamine. J. Agric. Food Chem., 2009, 57, 7194-7199.

6. Lee, J. E., Hong, Y. S. and Lee, C. H., Characterization of fermentative behaviors of lactic acid bacteria in grape wines through ${ }^{1} \mathrm{H}$ NMR- and GC-based metabolic profiling. J. Agric. Food Chem., 2009, 57, 4810-4817.

7. Li, C., Mu, L., Wang, J. Y., Lei, Z. H., Chen, J. Y. and Han, B. Z., Physiochemical and microbiological analysis of Fen-type Daqu. China Brew., 2009, 1(202), 140-142 (in Chinese).

8. Li, Z., Wang, N., Raghavan, G. S. V. and Vigneault, C., Volatiles evaluation and dielectric properties measurements of Chinese spirits for quality assessment. Food Bioprocess Technol., 2008. (doi: 10.1007/s11947-008-0162-y).

9. López-Rituerto, E., Cabredo, S., López, M., Avenoza, A., Busto, J. H. and Peregrina, J. M., A thorough study on the use of quantitative ${ }^{1} \mathrm{H}$ NMR in Rioja red wine fermentation processes. $J$. Agric. Food Chem., 2009, 57, 2112-2118.

10. Piveteau, P. G., Condon, S. and Cogan, T. M., Interactions between lactic and propionic acid bacteria. Lait, 1995, 75, 331343.

11. Schievano, E., Pasini, G., Cozzi, G. and Mammi, S., Identification of the production chain of Asiago d'Allevo cheese by nuclear magnetic resonance spectroscopy and principal component analysis. J. Agric. Food Chem., 2008, 56, 7208-7214.

12. Shi, J. H., Xiao, Y. P., Li, X. R., Ma, E. B., Du, X. W. and Quan, Z. X., Analyses of microbial consortia in the starter of Fen Liquor. Lett. Appl. Microbiol., 2009, 48, 478-485.

13. Son, H. S., Hwang, G. S., Kim, K. M., Kim, E. Y., Van den Berg, F., Park, W. M., Lee, C. H. and Hong, Y. S., ${ }^{1} \mathrm{H}$ NMRbased metabolomic approach for understanding the fermentation behaviors of wine yeast strains. Anal. Chem., 2009, 81, $1137-1145$

14. Wu, X. H., Zheng, X. W., Han, B. Z., Vervoort, J. and Nout, M. J. R., Characterization of Chinese liquor starter, "Daqu", by flavor type with ${ }^{1} \mathrm{H}$ NMR-based nontargeted analysis. J. Agric. Food Chem., 2009, 57, 11354-11359.

15. Xiong, Z. S., Research on three flavour type liquors in China (3) Fen flavour liquor - Xinghuacun. Liquor-making Sci. Technol., 2005, 7, 17-21 (in Chinese).

16. Yang, S. O., Kim, S. H., Cho, S., Lee, J., Kim, Y. S., Yun, S. S. and Choi, H. K., Classification of fermented soymilk during fermentation by ${ }^{1} \mathrm{H}$ NMR coupled with principal component analysis and elucidation of free-radical scavenging activities. Biosci. Biotechnol. Biochem., 2009, 73, 1184-1188.

17. Zhao, D., Li, Y. H. and Xiang, S. Q., Study on flavoring components of Daqu by headspace solid-phase microextraction gas chromatography-mass spectrometry (GC-MS). Liquor-making Sci. Technol., 2006, 5(143), 92-94 (in Chinese).

18. Zheng, X. W., Tabrizi, M. R., Nout, M. J. R. and Han, B. Z., Daqu - A traditional Chinese liquor fermentation starter. J. Inst. Brew., 2011, 117, 82-91.

(Manuscript accepted for publication November 2011) 